\title{
Die Wurzeln der Idiographischen Paläontologie: Karl Alfred von Zittels Praxis und sein Begriff des Fossils
}

\author{
Marco Tamborini
}

The Roots of Idiographic Paleontology: Karl Alfred von Zittel's Methodology and Conception of the Fossil Record

This paper examines Karl Alfred von Zittel's practice in order to uncover the roots of so-called idiographic paleontology. The great American paleontologist Stephen Jay Gould (1941-2002) defined the discipline of idiographic paleontology as illustration and description of the morphological features of extinct species. However, this approach does not investigate macroevolutionary patterns and processes. On the contrary, the paleobiological revolution of the 1970s implemented an epistemic methodology that illustrates macrovelutionary patterns and laws by combining idiographic data with a nomothetic form of explanation. This article elucidates the features of the idiographic data as well as the acquired knowledge coupled with this approach. First of all, Heinrich G. Bronn's (1800-1862) statistical method is analyzed. Zittel's practice arose as a reaction against the approximate conclusions reached by Bronn's quantitative approach. Second, the details of Zittel's methodology are described in order to bring out its peculiarities. The microscope played a pivotal role in creating and forming Zittel's morphological data. This analysis sheds new light on the reasons behind the so-called ideographic paleontology, thus revising Gould's historical reconstruction, as well as on the notion of paleontological data. However, even though Zittel aimed at reaching precise and stable conclusions, his data cannot be used for elucidating evolutionary mechanisms: they are scientific in a purely descriptive sense, but completely useless for biological investigations. Finally, this paper examines how Zittel's methodology affects the contemporary paleobiological enterprise and thereby reflects upon the notion of natural history.

Keywords: Paleontology, paleobiology, the fossil record, data, macroevolution

„Die Paläontologie hat längst aufgehört, sich als Lehre von den Leitfossilien ausschließlich in den Dienst der Geologie zu stellen. Sie ist allmählich zu einem selbständigen Zweig der biologischen Wissenschaften herangewachsen und nimmt an allen Bewegungen und Strömungen der letzten Theil" (Zittel 1897: 125). Mit diesen Worten eröffnete Karl Alfred von Zittel (1839-1904) 1894 den sechsten internationalen geologischen Kongress in Zürich. In diesem Jahr war Zittel schon ein anerkannter ${ }^{1}$ Paläontologe: Sein Meisterwerk, Handbuch der Palaeontologie (Zittel 1876a), war bereits gut zwanzig Jahre zuvor veröffentlicht worden. In diesem erfolgreichen, auch in anderen Sprachen 
erschienenen Werk, ${ }^{2}$ bearbeitete Zittel seinen Fossilbegriff: Die Aufgabe der Paläontologie sei es, sich mit ikonographischen, akkuraten und präzisen morphologischen Repräsentationen der ausgestorbenen und fossilisierten Tiere zu beschäftigen und damit wertvolle Daten für Darwins Abstammungslehre zu liefern.

Der sechste internationale geologische Kongress war daher die richtige Gelegenheit für Zittel, seine persönlichen Überzeugungen über den Status der Paläontologie einer breiteren Masse bekannt zu machen: Er war der Ansicht, dass die Paläontologie eine reife und selbstständige Wissenschaft sei, die einen wichtigen Zweig der Biologie bilde und ihre rein geologische und stratigrafische Periode überwunden habe. ${ }^{3}$ Anders als von Geologen und Stratigraphen postuliert, skizzierten Fossilien nicht mehr nur die Grundrisse von Ereignissen aus der geologischen Zeit, sondern seien vor allem Daten, die zur morphologischen und phylogenetischen Rekonstruktion sowie dem Studium der biologischen Funktion im Rahmen von Charles Darwins Abstammungslehre beitragen würden. Darüber hinaus arbeite der Paläontologe mit Mikroskopen und Werkzeugen, die bis ins kleinste Detail die morphologischen und physiologischen Strukturen der ausgestorbenen Organismen darstellen könnten - statt wie zuvor mit Tabellen und stratigraphischen Charts, um alles bis in die Tiefe der geologischen Zeit zu erfassen. Formen und physiologische Strukturen charakterisierten die Arbeit der Paläontologen, die nicht mehr unpräzise Entwicklungsgeschichten erzählen, sondern detaillierte Tatsachen für die Abstammungslehre sammeln wollten. Mit dieser Darstellung erfüllte Zittel Ernst Haeckels (1832-1919) Wunsch: Die Begründung einer nicht bloß empirischen, sondern auch theoretisch (durch Darwins Deszendenztheorie) angeleiteten Paläontologie. ${ }^{4}$

Mit dem vorliegenden Beitrag verfolge ich drei Ziele: Das erste Ziel ist es, im Zusammenhang mit Karl Alfred von Zittels Begriff des Fossils einige wichtige Momente der geschichtlichen Entwicklung der Paläontologie auf ihrem Weg in Richtung des „high table“ der Evolutionstheorie (Maynard Smith 1984) sowie deren Bedeutung zu thematisieren. Die Entwicklung der deutschen Paläontologie auf ihrem Weg zu makroevolutiven Theorien ab der Mitte des 19. Jahrhunderts wurde von mehreren Historikern entweder übersehen oder zu schnell beiseitegeschoben: ${ }^{5}$ Mit meiner Analyse möchte ich einen ersten Beitrag dazu leisten, diese große Lücke in der biologischen und paläontologischen Literatur zu schließen. Zittels Begriff des Fossils bietet eine bemerkenswerte Fallstudie, um die Grundlagen der Entwicklung der Paläontologie und Paläobiologie zu verstehen.

Einen wichtigen Moment in der geschichtlichen Entwicklung der Paläontologie bildet die sogenannte paläobiologische Revolution der 1960er und 1970er Jahre (vgl. Sepkoski and Ruse 2009) und sie macht auch das Endziel meiner historischen Analyse aus. ${ }^{6}$ Die Paläobiologie bietet einen neuen Zugang zur Fossilien, der den biologischen Wert der Paläontologie betont: 
Nach der paläobiologischen Revolution ist die Paläontologie eine rein biologische Wissenschaft, die einen grundlegenden Beitrag zur Evolutionstheorie leistet. Sie zielt darauf ab, biologische patterns und Prozesse, die nur durch Fossilien feststellbar sind, aufzuzeigen. Mit Hilfe der Datenbanken [wie z.B. Jack Sepkoskis Compendium of Fossil Marine Families (Sepkoski 1982)], statistischen Analysen von Daten und rechnerischen Simulationen untersuchen die Paläobiologen makroevolutive Phänomene und sind auf der Suche nach möglichen biologischen Erklärungen. Ein berühmtes Beispiel dafür ist die Theorie der Punctuated Equilibria ${ }^{7}$ der amerikanischen Paläobiologen Niles Eldredge (*1943) und Stephen J. Gould (1941-2002) (vgl. Eldredge/Gould 1972, Gould/Eldredge 1977).

Um dieses breitere evolutionäre Ziel zu erreichen und damit die Neuheit ihres Ansatzes zu betonen, schlugen die Paläobiologen eine historische Spaltung der paläontologischen Ansichten vor. Nach Wilhelm Windelband (1848-1915) griff der Paläobiologe Stephen J. Gould den Unterschied zwischen idiographischen und nomothetischen Wissenschaften auf. Unter den Begriff der „idiographischen Paläontologie“ fasste der amerikanische Paläobiologe eine Wissenschaft zusammen, die sich nur mit Einzelfällen beschäftigt und sich nicht für die Gesetzlichkeit interessiert, die diese Einzelfälle vereint. Zittels Praxis war nach Goulds Ansicht das beste Beispiel dafür: Sie strebt nach einer präzisen Repräsentation der einzelnen ausgestorbenen Tiere, ohne sich jedoch um die Gesetzmäßigkeit der dargestellten Phänomene zu kümmern.

Dagegen schlugen Gould und Kollegen eine paläobiologische Revolution vor, die die nomothetischen (also gesetzmäßigen) Aspekte der Paläontologie erneut ins Zentrum der paläobiologischen und biologischen Forschung brachte. Diese nomothetischen Komponenten ${ }^{8}$ stammten aus dem statistischen Lesen von Daten -, ein Vorgehen das Zittel mit Bestimmtheit ablehnte. Laut der Paläobiologen untersucht die Paläontologie - genauso wie die anderen schon von der modernen synthetischen Evolutionstheorie ${ }^{9}$ anerkannten biologischen Wissenschaften - die Gesetzmäßigkeit der Evolutionsphänomene, und ist damit eine nomothetische Disziplin.

Demnach wurde von verschiedenen zeitgenössischen Paläobiologen Zittels morphologischer und a-statischer Begriff der paläontologischen Daten zum Sündenbock für den verspäteten Auftritt der Paläontologie in der modernen Synthese gemacht. Das Problem dabei war - wie der amerikanische Paläobiologe Derek Ager (1923-1993) schrieb - dass, ,paleontology [is done] by the telephone book". Ager benutzte diesen Ausdruck für die Rezension von Ulrich Lehmanns Fossils Invertebrates (1983). Er bemerkte jedoch, dass Ulrich stark von Zittels Ansatz beeinflusst war: 
[T] his is a work of German scholarship; in fact it out-Zittels, Zittel, though at much shorter length: [...] apart from a brief section on the origin of life, there is very little on evolution, or paleoecology, or functional morphology, or geographical distribution or any of the dozen other subjects that many of us find so fascinating. No doubt that makes it the more scientific in a purely descriptive sense; it alsoregrettably - makes it extremely dull. (Ager 1983: 470)

Die Vertreter der paläobiologischen Revolution behaupten also, der Zittel'sche Begriff des Fossils sei auf einem substanziellen Problem gegründet: Mit dem Ziel, akkurate und echte Vorstellungen der ausgestorbenen Organismen herbeizuführen, habe er ein hohes epistemisches Niveau erreicht, allerdings besäße diese erreichte Ebene keinen Nutzen, um evolutive Mechanismen zu begreifen. Paläontologie sei lediglich wie ein Telefonbuch aufgebaut.

Das zweite Ziel dieses Aufsatzes ist es also, die Merkmale von Zittels Fossilbegriff zu beleuchten und damit sowohl ihre Voraussetzungen als auch die Wurzeln der sogenannten idiographischen Paläontologie (gegen die die paläobiologische Revolution der 1970er Jahre geführt wurde) zu erläutern. Als Ergebnis werde ich zeigen, dass die historische Entwicklung der Paläontologie auf ihrem Weg zur Evolutionstheorie komplexer ist als die von Gould vereinfachte Version. Zittel hatte gute Gründe dafür, die quantitativen und nomothetischen Aspekte zu meiden. Diese wurden zwar von Zittels Lehrer Heinrich G. Bronn (1800-1862) vertreten. Allerdings ergab Bronns quantitative Praxis nur subjektive Schlüsse und untergrub dementsprechend die Wissenschaftlichkeit der Paläontologie. Dagegen war Zittel stark davon überzeugt, dass eine ikonographische und akkurate Repräsentation der ausgestorbenen Tiere ermöglichen würde, die Paläontologie auf den sicheren Weg der Wissenschaftlichkeit zu bringen. Meine Arbeit thematisiert daher die Wechselpunkte eines epistemischen Wandels, und zwar zwischen der auf qualitativen Analysen gegründeten Zittel'schen Paläontologie und der so genannten paläobiologischen Revolution.

Das dritte Ziel dieses Aufsatzes ist die Historisierung der Eigenschaften der paläobiologischen Daten bzw. der Fossilien: Die folgenden Fallstudien stellen zwei verschiedene paläontologische Ansätze und Praktiken gegenüber, die auf unterschiedliche und gegensätzliche Weise den Begriff des Fossils prägten. Diese Arbeit ergänzt mittels einer Untersuchung der paläontologischen Praktiken Ende des 19. Jahrhunderts die paläobiologische „Naturgeschichte der Daten“ (Sepkoski 2013). Meine Untersuchung zeigt daher, was von Zittels Praxis am Ende in der paläobiologischen Revolution bleibt: Zittels Daten stecken das Feld ab, innerhalb dessen die paläobiologische Naturgeschichte der Daten durchgeführt werden kann.

Ich werde meinen Beitrag in zwei Abschnitte aufteilen: Im ersten Teil werde ich mich mit der statistischen Praxis von Heinrich G. Bronn auseinandersetzen. Zittel war Bronns Student in Heidelberg und dessen quantitative Methode beeinflusste Zittels idiographische Konzeptualisierung der 
Paläontologie daher stark. Im zweiten Teil werde ich der Praxis Zittels folgen, um die Strukturen seiner idiographischen Daten zu betonen. Nach Zittel wurden die paläontologischen Daten präzise morphologische Bildtatsachen, ${ }^{10}$ die Charles Darwins Abstammungslehre unterstützen und verstärken. Allerdings beschränkte dieser idiographische Begriff des Fossils den Vorgang des Wissensgewinns: Innerhalb dieser Kenntnis ist es dem Paläontologen nicht erlaubt, die makroevolutiven Mechanismen zu untersuchen.

Abschließend wende ich mich der paläobiologischen Revolution der 1970er Jahre zu. Sie wird zu einem ,indirekten quasi theoretischen Zugang“ (Valentine 1969: 266) zum Fossil führen, die der Deszendenztheorie Darwins den notwendigen theoretischen Stützpunkt gab, um die mangelhafte Natur des Fossils zu überwinden: Bekanntermaßen sind die Fossilien unvollkommen und unvollständig. Die Grundlage der Etablierung der Paläontologie als biologische Disziplin, wurde ins Zentrum der paläobiologischen Forschung der Zittel'sche Traum einer präzisen akkuraten und morphologischen Analyse gebracht: Die idiographischen Daten setzen die Grenze innerhalb derer die Gesetzlichkeit der Naturgeschichte gefunden werden kann.

\section{Die statistische Methode Bronns}

Zittel begann 1857 sein Studium der Medizin und Naturwissenschaft an der Ruprecht-Karls-Universität Heidelberg, wo er auf eine anregende Umgebung traf. Er studierte Paläontologie unter Heinrich Georg Bronn. Bronn war zu dieser Zeit der bekannteste Paläontologe Deutschlands und als einer der weltweit besten Naturwissenschaftler anerkannt: Seine Untersuchungen über die Entwickelungs-Gesetze der organischen Welt während der Bildungs-Zeit unserer Erd-Oberfläche (1858) gewannen den Preis der Académie des Sciences in Paris. Bronn interessierte sich für eine vielfältige Anzahl von Themen und Gebieten, er war einerseits Paläontologe, aber er nahm auch in der Zoologie, Paläogeographie, Kameralwissenschaft und Forstwissenschaft führende Positionen ein. Darüber hinaus war er der erste deutsche Übersetzer von Darwins Origin of Species (1859). Kurz: Der Heidelberger Paläontologe war „nicht nur der erste allgemeine Paläontologe, sondern [der] Wegbereiter der Abstammungslehre in derPaläontologie" (Quenstedt 1955).

Bronn prägte besonders Zittels Bildung - er war verantwortlich für seinen frühen Aufstieg in die Paläontologie - und beeinflusste auch die Auswahl seiner Methoden und Inhalte stark: Zittels Entscheidung für eine morphologische Methode war eine direkte Reaktion auf Bronns Ansatz. ${ }^{11}$

Bronns Ausgangpunkt ist der Begriff des Fossils, das ihm zufolge zwar ein unvollständiges Bild der Vergangenheit wiedergäbe, doch uns trotz dieser 
Unvollständigkeit gestatte, eine gewisse Kenntnis der Vergangenheit zu bekommen:

Die Erd-Rinde ist ein großes Buch; ihre Schichten sind die Blätter desselben, Versteinerungen die Buchstaben des Alphabets, womit es geschrieben, und der Inhalt ist die Geschichte der Schöpfung, von der uns kein lebender Augenzeuge Nachricht geben kann. Aber jene Blätter liegen unvollständig, zerrissen, durcheinander-geworfen und verblichen vor uns [...] Das Alphabet, worin das Buch geschrieben, war uns lange fremd [...] Wir bekommen daraus eine mehr und weniger vollständige Kenntnis von den Wesen, welche damals existierten, von ihrer Zahl und Organisation. (Bronn 1858: 75)

Heute ist uns das „Alphabet“ nicht mehr fremd - wir haben mittlerweile eine mehr oder weniger vollständige Kenntnis der ehemaligen Organismen, auf die die weitere biologisch-paläontologische Forschung aufbauen kann: Bronn suchte dafür nach einer Methode, um die damals existierenden Organismen und deren Zahl festzulegen. Im Vorwort zu der Naturgeschichte der drei Reiche schrieb Bronn: „es ist das erste Mal, dass der Versuch gemacht wird, eine Geschichte der gesamten Natur durch systematisches Ordnen und wissenschaftliche Beleuchtung rein thatsächlicher Beobachtungen ohne vorgefasste Theorie zu entwerfen" (Bischoff/Blum/Bronn/von Leonhard/Leuckart/Voigt 1841: VI). Die „rein thatsächlich[en] Beobachtungen“ stellte Bronn an den Objekten an, die er selbst im Laufe seines ganzen Lebens gesammelt, geordnet und schließlich in seinem Index Palaeontologicus (1848) veröffentlich hat. Jene Arbeit stellt alle gefundenen Tier-und Pflanzenfossilien in chronologischer (Nomenclator paleontologicus) und alphabetischer (Enumerator paleontologicus) Reihenfolge dar, und kann so die Grundlage für weitere Forschung, das heißt, für die Entdeckung der Gesetze der Natur der Welt liefern.

Die „rein thatsächlich[en] Beobachtungen“ sind insofern das, was heutzutage „systematisch-chronologisch-diagnostische Tabellen“ (Schumacher 1975: 77) genannt wird. Sie stellen die gefundenen Fossilien (z.B. die Klasse Cephalopoda, vgl. Abb. 1), in alphabetischer Reihenfolge und nach ihren Fundorten (Buchstaben a bis x) bzw. in dem Zeitraum, in dem sie lebten, dar (Sepkoski 2013, Tamborini 2015). Eine solche Aufstellung dient der Annäherung an ein sowohl biologisches als auch historisches Ziel: die Geschichte der organischen Wesen im Einzelnen zu erzählen (Bronn 1841-1849).

Dieses hohe Ziel kann jedoch nur erreicht werden, wenn der Sammlung der Fossilien eine mathematische Betrachtung derselben folgt: Bronn übernahm den Fachausdruck „Statik“ aus der Kameralwissenschaft und begründete damit die palaeontologische Statik. Diese Lehre besteht darin, „die Zahlen-Verhältnisse im Allgemeinen“ zu untersuchen, damit die Entwicklung der Organismen im Laufe der Erdentwicklung aufgezeigt werden könnte (Abb. 2).

Von Anfang an wurde Zittel also von der statistischen Methode Bronns stark beeinflusst: Die Heidelberger Zeit war bestimmend für seine weitere 


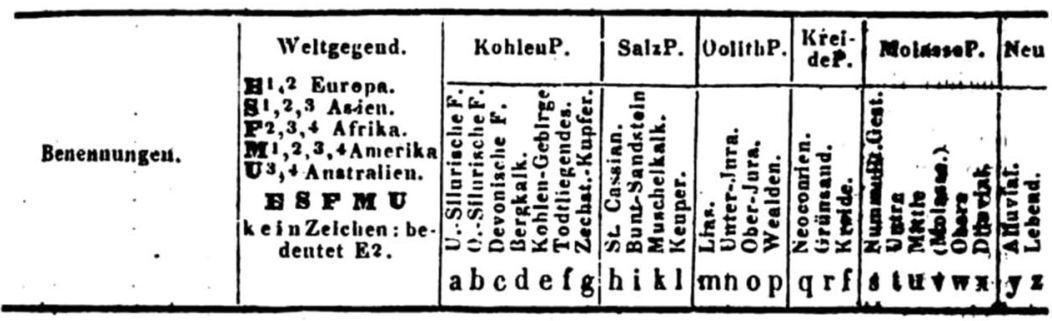

\section{Cl. XV. CephaL OPODA Cuv.: Kopffüsser.}

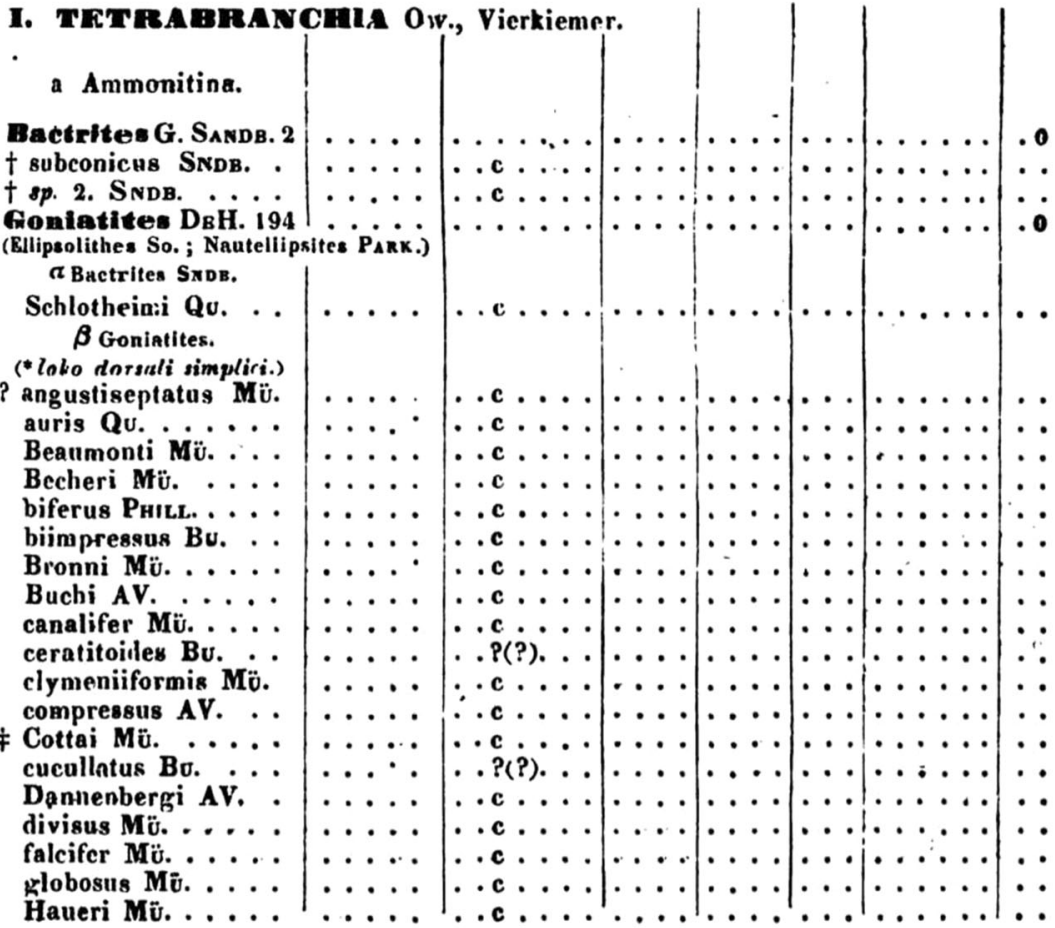

Abb. 1 Bronns stratigraphische und chronologische Tabelle (Bronn 1849b: 503)

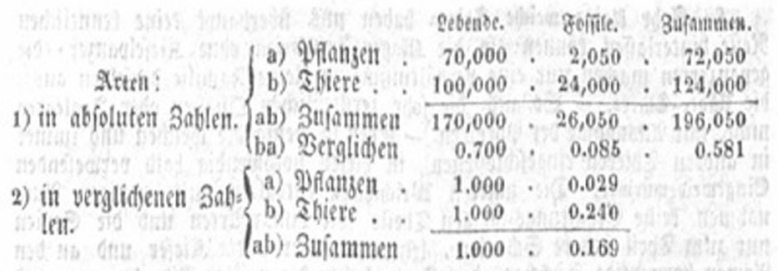

Abb. 2 Ein Beispiel für Bronns statistische Praxis (Bronn 1849b: 788) 
Entwicklung. Welchen epistemischen Status haben allerdings Bronns quantitative Ergebnisse? Bronn hatte hierauf eine sehr klare Antwort: Sie hätten keine notwendige und mathematische Gültigkeit und „,neue Beobachtungen können sie modifizieren oder umstoßen“ (Bronn 1849d: 809). Er setzte fort:

[W]enn wir auch die Resultate dieser Vergleichungen mit mathematisch scharfen Ausdrücken hinstellen, Diese dennoch nur annähernde ungefähre Werthe nach dem jetzigen augenblicklichen Stande unsrer Kenntnisse sind [...], dass das Bild, welches wir geben, sich auf die Summen der bisherigen Betrachtungen gründet, und dass neue Beobachtungen im Laufe der Jahre es sehr bedeutend umgestalten können, wenn auch viele Ergebnisse darin als für immer feststehend bereits betrachtet werden dürfen. (Bronn 1849a: 137)

Bronn behauptet, dass seine Ergebnisse nur einen annähernden ungefähren Wert hätten und deshalb eine sichere, exakte Erkenntnis nicht möglich sei. Die Tiefezeit spielt die Hauptrolle in dieser Indeterminierbarkeit der paläontologischen Gesetze: Nach Georges-Louis Leclerc Comte de Buffon (1707-1788) wird sie als sombre abîme bezeichnet. Das bedeutet, dass der Paläontologe nicht bestimmen kann, ,in wie ferne sich die den einzelnen Schichten, Formationen, Perioden entsprechenden Zeit-Abschnitte unter sich gleich verhalten; oder nicht der eine derselben 2-3-4-mal länger als der andere in gleiche Kategorie gestellte Abschnitt ist" (Bronn 1849a: 137). Obwohl Bronn die zeitliche Dimension durch die Regionen, in denen die Fossilien gefunden wurden, charakterisierte, war er mit dieser Methode nicht in der Lage, eine eindeutige zeitliche Dimension der Daten zu sichern: Bronn nahm an, dass die Buchstaben h, s, ü, v, in seiner stratigraphischen Tabellen (siehe Abb. 3) sich auf "kein[en] sicher[en] Platz" (ebd.: 2) bezögen.

Die Tiefezeit ist daher ein einschränkender Faktor: Da Bronns statistische Methode auf tabellarische Daten zurückgriff, die durch ihre Fundorten bestimmt waren, falls sich diese zeitlichen Erscheinungen paläontologisch nicht genau bestimmen ließen, ergaben die mathematischen Betrachtungen dieser Daten unpräzise und infundierte Ergebnisse. Da Bronns historische Gesetze insofern nur einen ungefähren Wert besitzen, riskiert die Paläontologie infolgedessen, ihren epistemischen Status innerhalb der biologischen Wissenschaften zu verlieren.

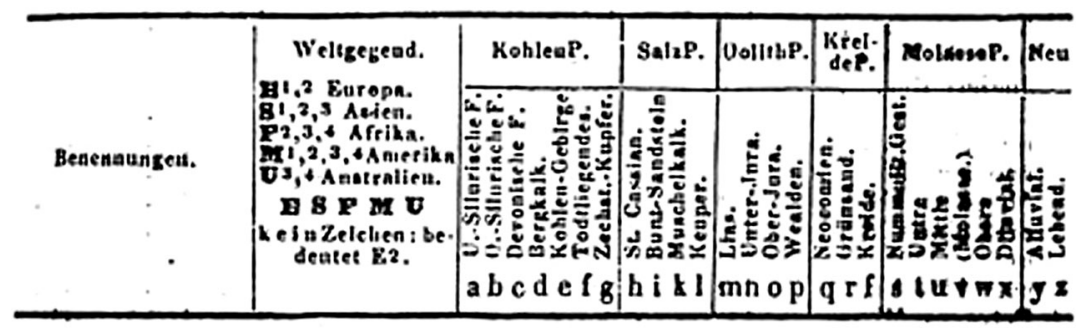

Abb. 3 Metadaten, die Bronn in seinen Tabellen nutzte (Bronn 1849b: 503) 
Zittels Begriff des Fossils war eine Reaktion auf Bronns quantitative Methode: um den biologischen Status der Paläontologie zu schützen, muss der Paläontologe diese quantitative Methode vermeiden. Aufgrund der Rolle der Zeit, soll der Paläontologe sich nicht mehr um die zeitliche Entwicklung der Lebewesen kümmern. Stattdessen soll er anfangen, gültige morphologische Daten für die Abstammungslehre zu sammeln. Fossilien müssen zu präzisen morphologischen Daten werden.

\section{Zittels Begriff des Fossils: von annähernden ungefähren zeitlichen zu präzisen morphologischen Daten}

Bronn war auf der Suche nach biostratigraphischen Tabellen, um die Zahlenverhältnisse zwischen den Fossilien im Allgemeinen berechnen zu können. Das Ziel von Bronns paläontologischer Statik war es, durch eine statistische Behandlung der Fossilien die Entwickelungs-Gesetze der organischen Welt zu bestimmen. Zittel lehnte die Darstellung der geologischen und paläontologischen Untersuchungen nach Bronn ab. Es ist gleichgültig, ob Bronn als der,,Wegbereiter der Abstammungslehre in der Paläontologie“ (Quenstedt 1955) betrachtet wurde oder das Stichwort „Paläontologie“ in der English Cyclopaedia (1870) Bronns Statistik in den Mittelpunkt stellte: Zittel war der Meinung, dass Bronns Methode zu riskanten Mutmaßungen führte und deshalb nicht verwendet werden sollte. Zittels Begriff des Fossils war demnach eine Reaktion auf die geringe Gewissheit in dieser Darstellung: Tabellen und quantitative Methoden erzielten nur unpräzise und dürftige Schlussfolgerungen. ${ }^{12}$ Die Paläontologie solle Fossilien weder als historische Dokumente behandeln noch mathematisch bearbeiten, da die epistemische und biologische Gültigkeit der paläontologischen Forschung aufgrund der spekulativen Ergebnisse zerstört werden könne. In Aus der Urzeit (1871) sagte Zittel darüber explizit: „leider steht jedoch gerade die Geologie, jene Wissenschaft, welche sich vorzugsweise mit der Entstehung und Entwicklung der Erde beschäftigt, ziemlich hülflos da, wenn es sich um Ermittlung der erste Zustande unseres Weltkörpers handelt" (Zittel 1871: 1). Die Paläontologie jedoch kann sich nicht erlauben, ,ziemlich hülflos“ zu sein, weil sie

die Wissenschaft von den Versteinerungen oder die Lehre von den alten Lebewesen [ist] [...]. Sie beschäftigt sich mit allen Fragen, welche die Eigenschaften, die systematische Stellung, die Verwandtschaft und Abstammung, die einstige Lebensweise, die räumliche Verbreitung und die zeitliche Aufeinanderfolge jener alten Wesen betreffen, sowie mit den Folgerungen, welche sich aus diesen Untersuchungen für die Entwickelungsgeschichte der Organismen und der Erde überhaupt ergeben. (Zittel 1876a: 1) 
Um die Wissenschaft von den Versteinerungen oder die Lehre von den alten Lebewesen zu bleiben, muss die Paläontologie positiv zur biologischen Entwickelungsgeschichte der Organismen beitragen. Dieses Ziel kann nur erfüllt werden, wenn der Paläontologe weder auf eine zweideutige statistische Methode noch einen irreführenden Begriff des Fossils zurückgreift. Der deutsche Historiker Karl Theodor von Heigel (1842-1915) kam zu demselben Ergebnis und schrieb in Bezug auf Zittels Lehre: „Man war der kosmologischmetaphysischen Spekulationen müde und setzte in die exakte Forschung die Hoffnung ad Natur". Paläontologie solle daher ,auf dem festen Boden der exakten Forschung”, durchgeführt werden, denn „eine Erklärung der letzten Ursachen der Dinge [...] entzieht sich der menschlichen Erkenntnis" (Heigel 1904: 7).

Zittels Ausgangspunkt war also folgender: Er war fest davon überzeugt, dass die wissenschaftliche Wahrheit ,ein wechselnder Begriff,, ${ }^{13}$ abhängig vom Umfang unseres Wissens [sei]. Was heute für wohlbegründete Wahrheit gilt, ist häufig nach wenigen Jahrzehnten als grober Irrtum erkannt" (Zittel 1902: 13) und folglich müssten die paläontologischen Untersuchungen einen festen Boden finden, auf den ihre Wissenschaftlichkeit gestellt werden könne.

Woraus besteht dann dieser feste Boden der Paläontologie? Diese Frage war nicht einfach zu beantworten, weil die Fossilien naturgemäß unvollständig und unvollkommen sind. Bronn versuchte diese Tatsache durch quantitative Methoden zu überwinden, ohne jedoch über die bereits erwähnten ungenauen Ergebnisse hinauszukommen. Zittel hingegen erkannte den "festen Boden der exakten Forschung" in der Begegnung zwischen Darwins Deszendenztheorie und dem neuen, durch die Anwendung des Mikroskops ${ }^{14}$ eröffneten Bereich der Morphologie. Die Deszendenztheorie garantierte der Paläontologie eine neue und ganz biologische Ebene: „Mit Darwin beginnt die neuste, moderne Periode für die Paläontologie" (Zittel 1899: 793). Die Deszendenztheorie lieferte einen Wendepunkt, „ebenso bedeutsam, wie jener, welcher sich an die Namen Werner, William Smith, Brongniart und Cuvier knüpft" (Zittel 1876a: 41). Die Paläontologie wurde schließlich eine biologische Wissenschaft, weil sie ,zahlreiche und gewichtige Beweise zu Gunsten der Descendenzlehre" lieferte. Sie brauchte Darwins Abstammungslehre, nicht weil sie einen evolutiven Mechanismus, sprich, die natürliche Auslese, hervorbrachte, sondern weil sie die Bedeutung der phylogenetischen Untersuchungen ins Zentrum der biologischen Forschung rückte. Insofern bot die Deszendenzlehre eine feste Grundlage für die Paläontologie, die ihr gestattete sich sowohl von den geologischen Untersuchungen als auch den hilflosen Spekulationen zu verabschieden: Paläontologie „hat vielmehr eine Fülle selbständiger Fragen zu lösen und in Gemeinschaft mit den übrigen biologischen Wissenschaften das Schöpfungsrätsel zu ergründen“ (Zittel 1899: 792). 
Um das Schöpfungsrätsel zu lösen, ohne sich auf gefährliche Spekulationen einzulassen, brauchte Zittel einen neuen Begriff der paläontologischen Daten, der mit der Deszendenzlehre zusammenpasste sowie eine neue paläontologische Praxis, die durch die Anwendung des Mikroskops stabile Ergebnisse erzielte.

Zittel fand seine Lösung schließlich in der Hauptaufgabe ${ }^{15}$ der Paläontologie: In der Einleitung der ersten Ausgabe seines Handbuches der Palaeontologie machte Zittel gerade darauf aufmerksam, dass die Hauptaufgabe der Paläontologie ,die Erzielung einer natürlichen, den morphologischen und phylogenetischen Erfahrungen entsprechende [...] Systematik" sei (Zittel 1876a: 1). Diese natürliche ${ }^{16}$ Systematik ließ sich auf den (paläo)zoologischen und (paläo)botanischen Bereich anwenden, weil „durch den Einfluß der Descendenztheorie de[r] Bruch zwischen den ausgestorbenen und noch jetzt lebenden Organismen fester geknüpft" sei. Es galt nun auszunutzen, dass die Paläontologie inzwischen über einen festen Platz in der Biologie verfügte: Der Paläontologe musste „Systematiker“ werden. Es gibt jedoch zwei Methoden anhand derer die Fossilien systematisiert werden konnten:

Man kann die Versteinerungen entweder in der Reihenfolge ihres Auftretens nach einzelnen Formationen oder Formationsabtheilungen aufzählen [à la Bronn], oder dieselben nach ihrem Bau, ihren morphologischen Eigenschaften, ihrer Entwicklungsgeschichte - kurz nach ihrer natürlichen Verwandtschaft an einander reihen. (Ebd.: 43)

Bei der ersten Methode erreicht man nur annähernde ungefähre Daten; während ,auf letzterem Wege [...] eine genaue Kenntniss der Fossilien hinsichtlich ihrer Organisationsverhältnisse, ihrer Beziehungen unter einander, sowie zu ihren jetzt lebenden Verwandten am sichersten und leichtesten zu gewinnen“ ist. Die paläontologische Praxis soll sich nach der zweiten Methode $^{17}$ richten: Paläontologische Daten sollten also nicht mehr als temporale Datensätze, in Tabellen dargestellt werden, sondern als morphologische und bildliche Tatsachen, die die Wirklichkeit der Lebewesen genau zu repräsentieren suchten, damit phylogenetische Rekonstruktionen gemacht werden könnten. ${ }^{18}$ Nur so könne die Paläontologie bedeutsame Tatsachen für die Deszendenzlehre liefern.

Die Versteinerungen sind jedoch meistens unvollkommen und unvollständig. Diese im paläontologischen Wissen klaffende Lücke konnte dank der Deszendenzlehre überwunden werden: Sie gab der Paläontologie ,einen Fingerzeig, wie ungefähr die noch fehlenden Formen aussehen müssen und wo [die Paläontologen] dieselben etwa zu suchen haben“ (ebd.: 42). Darwins Abstammungslehre bot daher der Paläontologie die feste Grundlage, die ihr gestattete sich weiterhin in den Bahnen der Biologie zu bewegen und darüber hinaus ein Werkzeug, um die Mängel ihrer epistemischen Daten zu beheben. Die paläontologische Praxis leitete daraus folgendes ab: 
In der Chemie construirt man häufig auf rein theoretischem Wege neue Verbindungen und stellt dieselben später durch Synthese dar. Ebenso ist die Palaeontologie im Stande die ehemalige Existenz von Formen vorauszusetzen und deren Organisation mit grosser Wahrscheinlichkeit zu vemuthen. (Ebd.: 42)

Die Paläontologen konnten daher rekonstruieren, was in ihren Daten fehlte: die komplette Struktur des ausgestorbenen Organismus. Die paläontologischen Daten spiegeln nicht nur die Merkmale der Versteinerungen wider, sondern sie korrigieren auch auf theoretische Art die mangelhafte Natur des repräsentierten Fossils und werden deshalb „wichtiger als der Gegenstand selbst" (Schlich 1995): Die paläontologischen Explananda werden die bearbeiteten und stabilen Bildtatsachen.

Es ist deshalb notwendig, Zittels Praxis genauer zu erklären, um diese Veränderung in dem Begriff der paläontologischen Daten zu verstehen. Ausgangspunkte sind folgende: einerseits muss der Paläontologe darauf verzichten, quantitative und tabellarische Daten $\mathrm{zu}$ benutzen, um den epistemischen Status der Paläontologie zu sichern; anderseits, da dank der Deszendenztheorie Darwins kein epistemischer Bruch zwischen der Paläontologie und Biologie besteht, unterscheidet sich die Methode der Untersuchung von Versteinerungen nicht von jener, welche der Zoologe oder Botaniker anwendet (vgl. Zittel 1895: 3). Der Paläontologe muss infolgedessen - genau wie andere Biologen (Nyhart 1987, 1995) - eine morphologische Methode verfolgen, um phylogenetische Untersuchungen durchzuführen. Durch diese Methode wurde die epistemische Sackgasse, verbunden mit der unvollständigen Natur des Fossils, überwunden:

Obwohl die Überreste der urweltlichen, als Versteinerungen überlieferten Lebewesen nur ein unvollständiges Bild darstellen, [...] lassen sie sich doch insgesamt in die großen Fachwerke der zoologischen und botanischen Systeme einfügen. Sie sind trotz aller Verschiedenheiten nach denselben Grundgesetzen gebaut wie die noch jetzt existierenden Organismen und ihre Bestimmung erfordert stets den sorgfältigen Vergleich mit demnächst verwandten lebenden Pflanzen und Tieren. (Zittel 1876a: 3)

Trotz aller Unterschiedlichkeiten kann der Paläontologe durch sorgfältigen Vergleich mit den am nächsten verwandten lebenden Pflanzen und Tieren wichtige Daten ermitteln, die zur Lösung des Schöpfungsrätsels beitragen.

Zittel schlug jedoch nicht ein bloßes Zurück zum heroischen Zeitalter der Paläontologie vor, in dem Geologen und Paläontologen à la Cuvier Fossilien ausgruben und klassifizierten, sondern strebte einen kompletten Wendepunkt in der paläontologischen Praxis an. Der Paläontologe Otto Max Johannes Jaekel (1863-1929) war Zittels Schüler und betonte explizit, dass beim Begriff der mikroskopischen Untersuchungen eine Unterscheidung getroffen werden müsse. Die Untersuchungen der Form des Lebewesens „fallen in das Gebiet der Morphologie und sind in der Palaeontologie fast ebenso alt als diese Wissenschaft selbst, da es nur auf diesem Wege möglich ist, kleine 
Organismen [...] zu erkennen“. Auf der anderen Seite gäbe es die Untersuchungen „des inneren geweblichen Aufbaues der Hartgebilde [...] welche in das Gebiet der Histologie fallen“ (Jaekel 1891: 179). Diese seien bedeutsamer, um Erkenntnisse über die Beziehung der fossilen Organismen untereinander zu erhalten.

Die Anwendung des Mikroskops unterschied so das moderne Zeitalter der Paläontologie von den morphologischen Untersuchungen Cuviers:

Es ist aber auch genötigt, aus den vorhandenen Resten so viel Belehrung zu ziehen, als nur immer möglich, und sieht sich darum nicht nur auf die äußerliche und makroskopische, sondern auch auf die feinere mikroskopische und histologische Untersuchung angewiesen (Zittel 1895: 3).

Das Mikroskop wurde benutzt, um die mikroskopische und histologische Struktur des ausgestorben Lebewesens $\mathrm{zu}$ beobachten und $\mathrm{zu}$ erforschen, damit wichtige Tatsachen für weitere phylogenetische Rekonstruktionen - die sogenannten Formenreihen ${ }^{19}$ (siehe Abb. 4) - innerhalb der Deszendenztheorie hervorgebracht werden konnten: „Keine grössere paläontologische Arbeit begnügt sich heutzutage damit, neue Formen zu beschreiben, mit den bisher bekannten zu vergleichen und ins System einzureihen"; sie fokussierten vielmehr auf die „Ermittlung der genetischen Beziehungen, der Abstammung, der Umbildung, der weiteren Entwicklung, kurz der Stammesgeschichte oder Phylogenie der untersuchten Organismen“ (Zittel 1897: 125).

Wie in der Biologie eröffnete die Anwendung des Mikroskops ein neues und bis zu diesem Moment noch unbekanntes Forschungsgebiet (Wilson 1995, Breidenmoser/Engler/Jirikowski/Pohl/Weiss 2010). Aber im Unterschied zur Biologie sei „,in manchen Abteilungen des Pflanzen- und Tierreichs die Paläontologie durch ausgiebige Verwertung histologischer Merkmale der Botanik und Zoologie vorausgeeilt" (Zittel 1895: 3). Diese Überwindung markierte die Entstehung der idiographischen Daten der Paläontologie, die nahezu vollständige Organismen sichtbar machen können, welche die Grundlage für eine komplette und präzise Beschreibung von Einzelfossilien liefern. Die idiographischen Daten boten den Paläontologen nicht die Möglichkeit ihrer Vorstellungskraft freien Lauf zu lassen, sondern präzise und akkurate Regeln zum Sammeln von Tatsachen und Beweisen. In ihrem Streben nach Präzision und Gründlichkeit lässt diese Praxis keinen Platz für die Ermittlung breiter evolutiver Mechanismen (Tamborini Under Review).

Die fünfte Abbildung repräsentiert die Familie der Coeloptychidae Zittel. Sie ist hilfreich, um die Entstehung von idiographischen Daten genauer zu analysieren (siehe Abb. 5).

Diese Familie der Schwämme ist erstmals während der 1860er Jahre von Zittel beschrieben worden: Nur dank der Anwendung des Mikroskops war Zittel in der Lage, neue Merkmale dieser Familie wahrzunehmen, sie zu klassifizieren und schließlich zu reproduzieren. Zittel nahm an, dass eine 

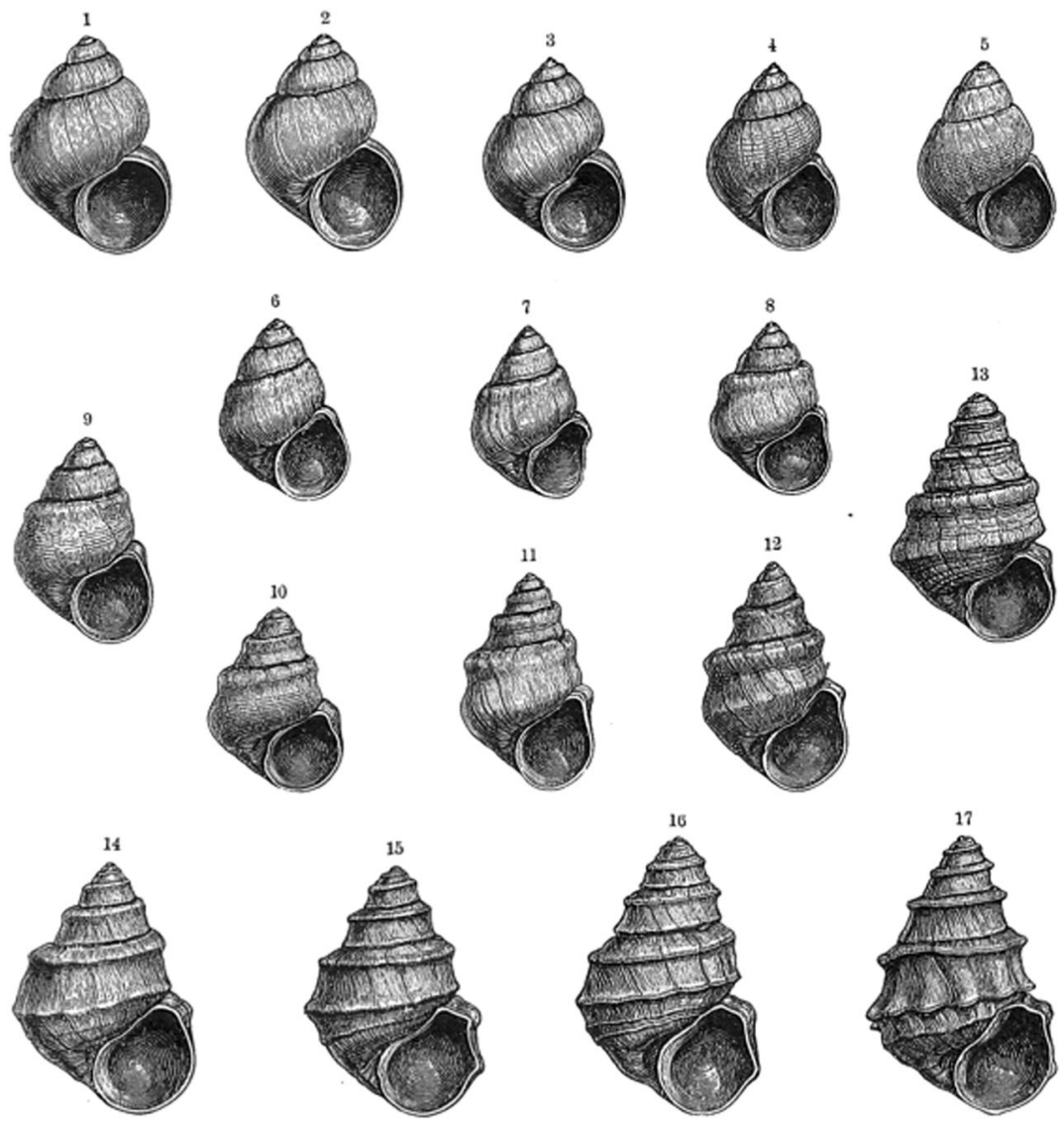

Abb. 4 Melchior Neumayrs Formenreihe, die allmähliche und schrittweise Veränderung der Organismen zeigt (Neumayr 1895: 16)

genaue Kenntnis, die auf dem aktuellen Stand basiere, für die phylogenetische Entwicklung dieser Familie unmöglich sei, denn ,[o]bwohl bei den lebenden Spongien schon längst der feinere, mikroskopische Skeletbau fast ausschliesslich in der Systematik Verwerthung fand, hielt sich bei den fossilen lediglich an die oberflächlichsten Merkmale der äusseren Formerscheinung“" (Zittel 1876b: 4). Die phylogenetische Kenntnis könne nicht auf ungefähre oberflächlichste Merkmale der äußeren Formerscheinung gestützt werden, im Gegenteil sollten die phylogenetischen Beziehungen ,erst durch eine umfassende mikroskopische Untersuchung der verschiedenartigen, in fossilem Zustande vorliegenden Skelete und durch ein genaueres Studium des physiologisch so wichtigen Canalsystems" analysiert werden. Nur dadurch würde es gelingen, „den sicheren Nachweis“ für die Abstammungslehre Darwins zu gewinnen. 
5. Fanilie. Coeloptychidae Zitt.

Schirm- oder pilzförmig gestielt. Wand dünn, mäandrisch gefaltet. Falten radial angeordnet, gegen den Aussenrand des Schirms gegabelt, auf der Unterseite unbedeckt. Seitenwand und Oberfläche des Schirms von einer porösen Deckschicht ïberspannt, welche die Falten vollständig verhiullt. Kanalostien auf den Faltenrïcken der Unterseiten. Skelett sehr regelmï̈sig, die Kreuzungsknoten durchbohrt, die Arme der Sechsstrahler mit feinen, stachligen Fortsätzen.
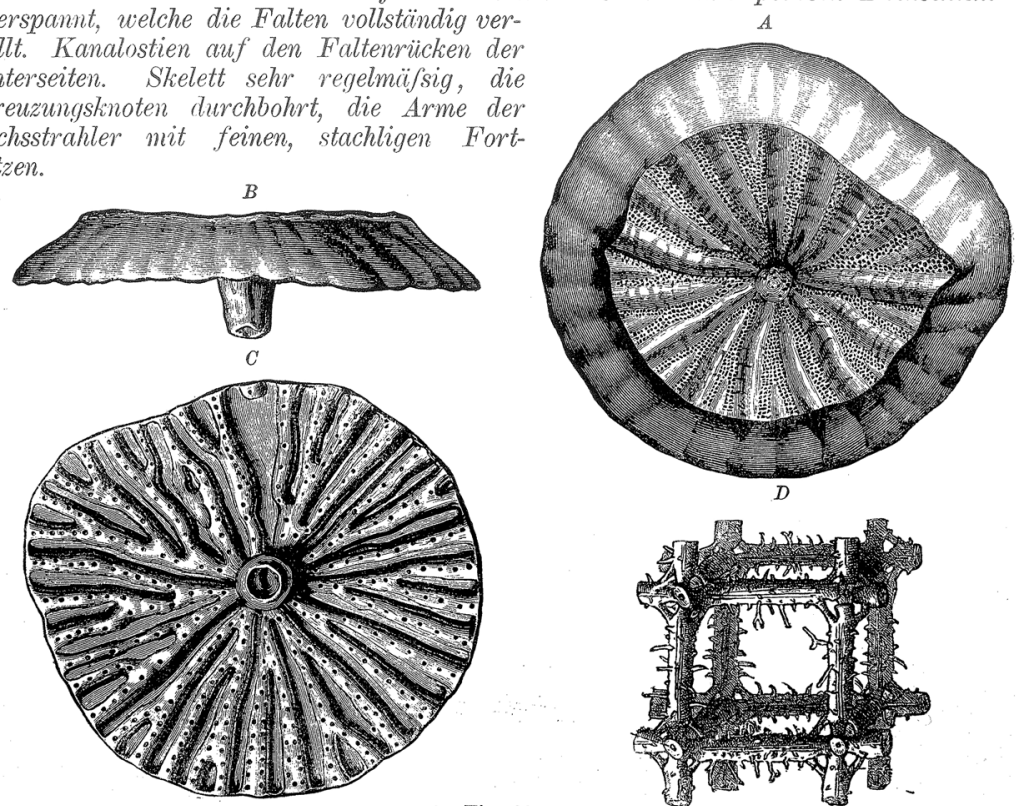

Fig. 80.

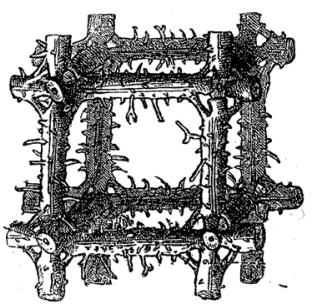

Coeloptychium agaricoides Goldf. Ob. Kreide. Vordori bei Braunschweig. $A$ Von oben. $B$ Von der Seite. $C$ Von unten $2 / 3$ nat. Gröfse. $D$ Skelett $6 \%{ }_{1}$.

Einzige Gattung Coeloptychium Goldf. (Fig. 80) in der oberen Kreide von Norddeutschland, England, Südruf́sland.

\section{Familie. Maeandrospongidae Zitt.}

Schucammliörper aus dünnwandigen, vielfach verschlungenen und teilweise veruachsenen Röhren oder Blättern zusammengesetzt, welche knollige, birnförmige, becherförmige oder strauchartig verästelte Stöcke bilden. Zwischen den Röhren bleiben grö/sere Löcher oder Zwischenrë̈ume frei, die ein sogenanntes Interkanalsystem bilden. Eigentliche Kanäle kaum entwickelt. Deckschicht fehlend oder eine zusammenhängende Kieselhaut auf der Oberflüche bildend.
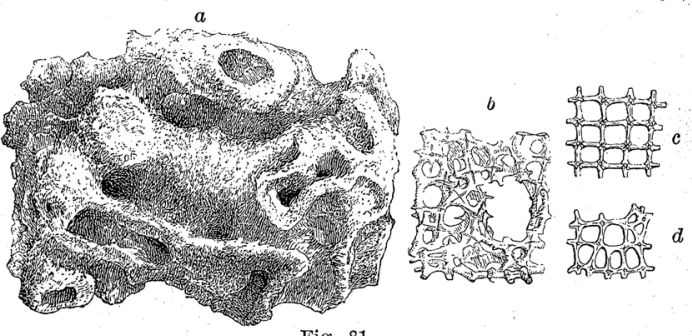

In der Kreidehäufig;

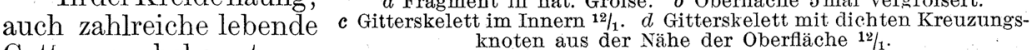
Gattungen bekannt.

Plocoscyphia Reufs. (Fig. 81). Knollige, kuglige, aus mäandrisch gewundenen, anastomosierenden Röhren oder Blättern bestehende Stöcke.

Abb. 5 Das Stichwort Coeloptychidae Zittel in Zittels Grundzüge der Palaeontologie (Zittel 1895: 59) 
Die paläontologische Praxis bestand also darin, dass die ausgegrabenen Schwämme mit Hilfe des Mikroskops und einem Präparationsmittel wie etwa Salzsäure, das den kompakten Schirm des Coeloptychium auflöst, systematisiert wurden und die repräsentierten Bildtatsachen auf diesem Wege eine biologische Bedeutung bekommen konnten. Bei Anwendung richtiger Präparation und Instrumente gelang es dem Paläontologen bisher unbeobachtete morphologische Merkmale der ausgestorbenen Organismen hervorzuheben: Diese konnten dann als sichere Beweise für weitere evolutive Untersuchungen angeführt werden und erhielten damit zoologische Gültigkeit.

Zittels Praxis betont also eine wichtige Veränderung in der paläontologischen Forschung: erstens, nicht Hammer, stratigraphische Tabellen und Charts, sondern chemische Präparationen, geeignete Lupen und die Camera lucida werden die neuen paläontologischen Instrumente. Zweitens, die paläontologischen Daten bestehen aus detaillierten anatomischen, physiologischen und morphologischen Untersuchungen, die für phylogenetische Analysen verwendet wurden, um die Deszendenzlehre Darwins zu bekräftigen. Drittens, die paläontologischen Bilder schaffen Bildtatsachen: Sie reproduzieren nicht nur die ausgestorbenen Lebewesen, sondern korrigieren auch ihre mangelhafte Natur und machen ihre verlorene Gestalt wieder sichtbar. Zu dieser wiedererlangten Sichtbarkeit trug auch die zentrale Rolle bei, die diese Abbildungen in Zittels Praxis einnahmen: Obwohl die Bilder seit Anfang des 18. Jahrhunderts eine zentrale Rolle in der paläontologischen Forschung und Literatur spielten, waren diese oft nur in den Anhang von Textbüchern oder Aufsätzen eingefügt: So stellte beispielsweise der deutsche Paläontologe Friedrich August von Quenstedt (1809-1889) im Handbuch der Petrefaktenkunde die Abbildungen der von ihm beschrieben Fossilien in den Anhang seines 1239-seitigen Handbuchs. Vor Zittel waren die textlichen Beschreibungen von Fossilien und nicht etwa ihre Abbildungen die wichtigsten Elemente der paläontologischen Forschung. Diese Praxis fußte auf der Überlieferung der Naturgeschichte, die sich ab Mitte des 18. Jahrhunderts etabliert hatte. ${ }^{20}$ Zittel hingegen platzierte die Bilder in der Mitte seines Handbuches. Sie bekamen einen neuen und zentraleren Platz, weil sie endlich objektive Szenen aus der Tiefenzeit abbildeten (Rudwick 1985). ${ }^{21}$

\section{Idiographische Daten und „,ein indirekter quasi theoretischer Zugang'"}

Seit Ende des 18. Jahrhunderts beschäftigte die Paläontologen eine neue, zentrale Frage: Inwiefern untergräbt die mangelhafte Natur des Fossils die paläontologische Kenntnis? In diesem Aufsatz habe ich die Genese der 
idiographischen Paläontologie aufgezeigt - eine Praxis, die auf eine sichere und objektive paläontologische Kenntnis abzielt. Ich habe argumentiert, dass Zittel, um diesen Zweck zu erreichen, einen morphologischen Fossilbegriff vertrat, der aus präzisen morphologischen Bildtatsachen statt aus annähernden ungefähren zeitlichen Datensätzen bestand. Zittel lehnte Bronns quantitative Methode ab und bediente sich des Mikroskops im Bemühen die mangelhafte Natur des epistemischen Dings der Paläontologie zu überwinden, ohne die biologische Grundlage von Darwins Abstammungslehre zu verlieren.

Das Ergebnis rechtfertigte Zittels Praxis, denn es gelang ihm, die unvollständige und unvollkommene Natur des Fossils zu überwinden: Unter dem Stichwort „Coeloptychidae Zittel" finden wir in Grundzüge der Paläontologie ein akkurates und vollständiges Bild der ausgestorbenen Spongienfamilie. Dank den mikroskopischen Beobachtungen einerseits und der paläontologischen Fähigkeit, diese korrekt zu repräsentieren andrerseits erhalten die paläontologischen Daten einen neuen epistemischen Status: Sie sind die detaillierten und vollständigen Bilder, die sich in Lehrbüchern, Katalogen oder Zeitschriften $^{22}$ finden.

Die eigentliche Frage lautet daher, wie eine Naturgeschichte, die eine verlässliche biologische Kenntnis anstrebt, geschrieben werden kann. Sowohl Bronn als auch Zittel wandten eine vergleichende Praxis an, die in den naturhistorischen Untersuchungen seit langem gesetzt war (Farber 2000, Larson 1994, Lepenies 1976). Bronn suchte nach einer Methode, um die in Tabellen präsentierten komparativen Beobachtungen $\mathrm{zu}$ analysieren: Seine mathematische Betrachtung der Fossilen, sprich, die paläontologische Statik, zielte darauf ab, die Entwicklung der Organismen im Laufe der Erdentwicklung aufzuzeigen. Allerdings erzielte er trotz der mathematischen Betrachtung der Daten Folgen und Gesetze, die „annähernde ungefähre Werthe" haben (Bronn 1849a: 137): In der unpräzisen und unbegründeten Historisierung seiner Daten übersah er die zerstörerische Rolle der tiefen Zeit.

Zittel verfolgte dagegen eine radikalere mechanische Objektivität (Daston/Galison 1992, 2007, Porter 1995), die die Grundregeln der paläontologischen Praxis vorgaben. Er vertrat diese Objektivität jedoch nicht, weil die Angst vor dem schwindelerregenden Fortschritt sein eigenes Selbst beherrschte (Daston 1998), sondern weil er begriff, dass sogar die wilde Vorstellungskraft ihre Macht im Abgrund der geologischen Zeit verliert: ${ }^{23}$ Wenn die Paläontologie über gültige, objektive und biologische Daten verfügen möchte, dann muss die Naturgeschichte a-temporal werden. Die Fossilien besitzen einen unschätzbaren Wert, wenn sie anhand ihrer morphologischen Eigenschaften in eine natürliche Systematik eingeordnet werden können und damit unabhängig von der zeitlichen Dimension betrachtet werden. Das impliziert, dass sich zwischen den Fossilien, die als historische Dokumente in Tabellen systematisiert sind, und den Fossilien, die als paläontologische Daten genutzt wurden, ein Bruch vollzieht: 
Die Versteinerungen sind in diesem Werke [Zittels Grundzüge] vorzugsweise als fossile Organismen behandelt, während ihre Bedeutung als historische Dokumente zur Altersbestimmung der Erdschichten nur in zweiter Linie Berücksichtigung finden konnte. (Zittel 1895: IV)

Die fossile[n] Organismen sind die neuen paläontologischen Daten, während ihre Bedeutung als historische Dokumente zur Altersbestimmung der Erdschichten nicht mehr zur paläontologischen Hauptaufgabe gehört.

Dies führte einen Wandel in der paläontologischen Literatur herbei: „Der Charakter der paläontologischen Literatur hat sich demgemäß auch nicht unerheblich geändert; die reine stratigraphisch-paläontologischen Arbeiten scheiden sich immer schärfer von den biologisch-systematischen, und die letzteren betonen mehr und mehr die geologische Richtung" (Zittel 1899: 793). Die paläontologische Literatur betont die morphologische und bildliche Natur ihrer Daten und beseitigt Tabellen und Charts: Bis zur paläobiologischen Revolution der 1960er und 1970er Jahre waren die wichtigsten paläontologischen Handbücher und Textbücher (wie z.B. Romer 1933, Schrock/Twenhofel 1953) danach ausgerichtet, die Fossilien morphologisch zu repräsentieren.

Andererseits implizierte die Behandlung von Fossilien als Organismen die Entstehung und Konsolidierung einer Disziplin, die nicht zu annähernden, ungefähren Ergebnissen, sondern zu präzisen morphologischen und biologischen Tatsachen kommt:

Welche Wunder erschloss das Mikroskop in kurzer Zeit den fleissigen Forschern! [...] man [hatte] nun nicht mehr bloss Ahnungen und Hypothesen, sondern Beweise. Die herrliche Folge immer vollkommener werdender Formen und Funktionen war nicht mehr dichterische Intuition, sondern eine Tatsache für den gesunden Menschenverstand. (Heigel 1904: 6)

Die dichterische Darstellung der Paläontologie machte Platz für eine präzise, obgleich trockene Naturgeschichte, die auf der Suche nach akkuraten Bildtatsachen war. Allerdings führte diese Suche in eine epistemische Sackgasse. 1980 bemerkte Gould dazu, dass die Paläontologie sich in einer ähnlichen Lage wie die Psychologie am Ende des 19. Jahrhunderts befände:

A string of raw facts; a little gossip and wrangle about opinions; a little classification and generalization on the mere descriptive level; [...] not a single law in the sense in which physics shows us laws, not a single proposition from which any consequence can causally be deduced. This is no science, it is only the hope of a science (Gould 1980: 96).

Die Suche nach stabilen Tatsachen beschränkte die paläontologische Untersuchung auf präzise morphologische und phylogenetische Analysen innerhalb von Darwins Theorie. Nach Zittels Unternehmen ist es alleiniges Aufgabengebiet des Paläontologen, die Gültigkeit von Darwins Mechanismen zu beweisen. Die Paläontologie ist nichts anderes als eine nachrangige Disziplin, an die die makroevolutive Ebene angeschlossen ist. Paradoxerweise schränkten 
die Grundregeln der Zittel'schen Naturgeschichte den epistemischen Raum der Paläontologie ein. Die mechanische Objektivität, die eine wichtige Rolle auf anderen wissenschaftlichen Gebieten spielte, hatte eine beschränkende Funktion in der Paläontologie: Obwohl sie prima facie die biologische Veranlagung der Paläontologie sicherte, gelang es ihr nicht, die Entwicklungsgesetze der Lebewesen in der geologischen Zeit hervorzubringen.

Die paläobiologische Revolution der 1960er und 1970er Jahre stellte einen neuen Begriff von Daten und Naturgeschichte vor, die sich nicht auf einer mechanischen Objektivität begründete, sondern auf einer Kombination von nomothetischen und idiographischen Elementen, die von der Kontingenznatur der Geschichte geprägt sind. Stephen J. Gould, Jack Sepkoski, Normann Newell (1909-2005), James Valentine (*1926) und die anderen Verfechter der amerikanischen paläobiologischen Revolution praktizierten ,einen indirekten quasi theoretischen Zugang" (Valentine 1969) zu den Fossilien: Er bestand aus einem "generalized rereading“ der Fossilien ,that involved seeking generalizations and producing models based on a careful tabulation and statistical analysis" (David Sepkoski 2012: 383). Die paläontologischen idiographischen Daten sind nicht mehr einzelne morphologische in Bildern dargestellte Entitäten (à la Zittel), sondern Zeichenfolgen, die in Datenbanken und Tabellen eingestellt und in Grafiken dargestellt wurden, wie etwa Jack Sepkoskis Compendium of fossil marine families (Sepkoski 1982). In diesem Kompendium klassifizierte Jack Sepkoski 4.075 Fossilienfamilien von Meerestieren nach ihrem ersten und letzten Auftreten.

Obwohl auch die Paläobiologen die Fossilien klassifizieren, ist diese Klassifikation als Ausgangpunkt für weitere biologische Schlüsse, die evolutive Mechanismen gründlich analysieren, gedacht: Wie Jack Sepkoski schrieb

[I]n the letter of submission [associated with the compendium] I wrote that the data could constitute a basis for studies of "mass extinction, evolutionary rates, and patterns of diversification". Diversity - the very reason the data based existed-had been relegated to last place. (Sepkoski 1994: 140)

Die klassifizierten Fossilien haben daher eine breitere Bedeutung: Sie werden als Basis für evolutive Studien, die auf der Suche nach den nomothetischen Elementen der Phänomene sind, genutzt.

Mit Hilfe von statistischen Analysen untersucht die paläobiologische Praxis diese klassifizierten Daten und identifiziert demnach das Fossil mit einer Stichprobe, die statistisch untersucht und tabellarisch sowie grafisch dargestellt werden kann: „the graph provides the raw material from which evolutionary rates can be calculated" (Raup/Stanley 1971: 268).

Diese Praxis veranlasste eine paläobiologische Naturgeschichte der Daten, die die Gesetzlichkeit sowie die Kontingenz der Naturgeschichte offenlegt. So war beispielsweise Jack Sepkoski in der Lage sowohl anhand der von ihm klassifizierten Daten als auch deren statistischer Bearbeitung die Diversität des 
Phanerozoikums als drei gekoppelte logistische Funktionen zu beschreiben (Sepkoski 1978). Es ist ein bedeutendes Beispiel für die paläobiologische Kombination von idiographischen und nomothetischen Ansätzen. So beschrieb auch Gould Sepkoskis naturhistorische Untersuchung (Sepkoski 1978):

Here we see an interesting and fruitful interaction of nomothetics and idiographics. The form of the model remains nomothetic - the "real" pattern arises as an interaction between two general curves of the same form, but with different parameters. Idiographic factors determine the parameters and then enter as boundary conditions into a nomothetic model. (Gould 1980: 115)

Trotz der nomothetischen Form von Sepkoskis Untersuchung, die die Gesetzlichkeit der Entwicklung, beispielsweise der Organismen während des Phanerozoikums, mittels einer mathematischen Funktion ausdrückt, bestimmen die in den Datenbanken eingestellten idiographischen Daten die Randbedingungen, die von der „Kontingenznatur der Geschichte“ (Gould 1989) beeinflusst sind. Die paläobiologische Naturgeschichte ist daher das Zusammentreffen von idiographischen Datenbankdaten mit statistischer und mathematischer Struktur.

Die paläobiologische Naturgeschichte der Daten verwirklicht jedoch Zittels Traum einer präzisen, objektiven und akkuraten Paläontologie: Die morphologische Analyse der Kontingenznatur der Daten ermöglicht die Festlegung der Randbedingungen mittels derer die Gesetze der Naturgeschichte und dementsprechend auch eine wohlbegründete und biologisch orientierte Paläontologie entwickelt werden konnte. Die in den idiographischen Daten verkörperten Randbedingungen spielen deshalb eine zentrale epistemische Rolle: Sie können die Struktur und die Gültigkeit makroevolutiver Gesetze ändern, weil Kontingenz das Wesen der Geschichte ist (Gould 1989).

\section{Danksagung}

Hiermit möchte ich mich bei Heike Lehner und Michael Bühler (Bibliothek Geowissenschaften Heidelberger Universität) für ihre großartige Hilfe bei der Suche nach bibliographischen Materialien bedanken. Darüber hinaus bedanke ich mich bei Johannes Seidl, David Sepkoski und Peter McLaughlin. Mein besonderer Dank gilt Verena Rith.

\section{Anmerkungen}

1 Die Bedeutung Karl Alfred von Zittels für die Paläontologie ist von einem solchen Ausmaß, dass ihn seine Schüler ,begeistert [...], mit Überzeugung und neidlos auch die 
deutschen, englischen und amerikanische Fachgenossen den ersten Paläontologen der Gegenwart" nennen (Heigel 1904: 8). Zittels Begriff des Fossils war äußerst erfolgreich: zwischen Ende des 19. Jahrhunderts und Mitte des 20. Jahrhunderts war er für vielen Paläontologen, wie etwa den Briten Henry Alleyne Nicholson (1844-1899), den deutschen Melchior Neumayr (1845-1890) oder den amerikanischen Alfred Romer (1894-1973), der Prototyp für die paläontologische Forschung. Wie der amerikanische Paläontologe Henry Fairfield Osborn (1857-1935) zu Beginn des 20. Jahrhunderts berichtete, wurde die Münchner Universität unter Zittel, "the Mecca of paleontologists, young and old" (Osborn 1904: 186).

2 Das Handbuch der Palaeontologie wurde ins Englische und Französische übersetzt und somit in die Sprachen, die für die damalige Paläontologie am bedeutendsten waren.

3 Paläontologie wurde als Hilfswissenschaft der Geologie betrachtet: Der wissenschaftliche Wert der Fossilien bestand vor allem in ihrer Funktion als Zeitmarken. Vgl. Quenstedt 1852, 1858, Oppel 1856-1858.

4. „Wenn wir die Summe aller Organismen, welche von einer und derselben einfachsten, spontan entstandenen Stammform ihren gemeinschaftlichen Ursprung ableiten, als einen organischen Stamm oder Phylon bezeichnen, so können wir demnach die Palaeontologie die Entwicklungsgeschichte der Stämme oder Phylogenie nennen. Allerdings existirt [sic!] die Palaeontologie in diesem Sinne noch kaum als Wissenschaft; und erst nachdem durch Darwin die Abstammungslehre neu begründet war, haben in den letzten Jahren einige Palaeontologen angefangen, hier und da den genealogischen Massstab an die palaeontologischen Entwickelungsreihen anzulegen, und in der Formen-Aehnlichkeit der nach einander auftretenden Arten ihre wirkliche Blutsverwandtschaft zu erkennen. Wir können aber nicht daran zweifeln, dass dieser kaum erst emporgekeimte Samen sich rasch zu einem gewaltigen Baume entwickeln wird, dessen Krone bald eine ganze Reihe von anderen wissenschaftlichen Disciplinen in ihren Schatten aufnehmen und überdecken wird." (Haeckel 1866: 59)

5 Siehe z.B. Rudwick 2005, 2008, Beringer 1954, Wagenbreth 1999, Ziegler 1984, Hölder 1989, Greene 1982. Ausnahmen davon Sepkoski/Ruse 2009, Sepkoski 2012, Reif 1986, Tamborini 2015.

6 Über den Unterschied zwischen Paläontologie und Paläobiologie siehe Sepkoski 2012, Sepkoski/Ruse 2009, Turner 2011.

7 Laut dieser Theorie besteht das Phänomen der evolutionären Speziation aus einer Phase von Stasi (Stillstand) und einer Phase von plötzlichen und schnellen Veränderungen. Diese Theorie war so erfolgreich, dass sie auch in andere nicht-biologische Bereiche, wie etwa die Linguistik, übertragen wurde.

8 Beispielsweise Eldredges und Goulds Punctuated Equilibria (Eldredge/Gould 1972), Jack Sepkoskis Kurve der evolutiven Faunen (Sepkoski 1978, Sepkoski/Bambach/Raup/ Valentine 1981) sowie David Raups und Jack Sepkokis Theorie der Periodizität des Massenaussterben (Raup/Sepkoski 1984).

9 Unter der modernen synthetischen Evolutionstheorie versteht man die Vereinigung in den 1930er Jahren von der Darwinistischen Evolutionstheorie mit den Ansichten und Daten aus der Genetik, Ökologie und der biologischen Systematik.

10 Wie ich durch die Analyse von Zittels Praxis zeigen werde, werden die paläontologischen Daten, bzw. die Fossilien, akkurate Repräsentationen. Durch ihre Abbildhaftigkeit und ihre morphologische Präzision werden sie zu Bildtatsachen. Dieser Zusammenhang ermöglicht neue und wichtige Fragestellungen über den Begriff der paläontologischen Repräsentation und Sichtbarkeit, auf die in diesem Aufsatz nur hingewiesen werden kann, beispielsweise: Welchen Unterschied gibt es zwischen dieser Art von Visualisierung und der von anderen naturhistorischen Wissenschaften? Was lösten diese Visualisierungen aus? Zum Begriff der Bildtatsache siehe beispielsweise Heumann/Hüntelmann 2013, zum Begriff der wissenschaftlichen Repräsentation siehe unter anderem Daston 2014, Rheinberger/Hagner/Schmidt-Wahrig 1997, Golinski 1998 und zur Visualisierung Hentschel 2014, Kulvicki 2010, Dupré 2010, Crary 1988, Blum 1993.

11 Das zeigt, dass dieser morphologische Ansatz in Deutschland und den USA eine unterschiedliche Genese durchlief. zur Entwicklung dieser Praxis in den USA siehe Rainger 1981, 1991. 
12 Auch Ernst Haeckel teilte diese Meinung: Er hielt Bronns statistische Ergebnisse für dürftig (Haeckel 1866).

13 Die Anerkennung eines wechselnden Wahrheitsbegriffs hat seine Wurzeln sowohl konkret in Zittels Bildung als auch im wissenschaftlichen Milieu des auslaufenden 19. Jahrhunderts: viele Philosophen sowie Wissenschaftler erkannten die anti-metaphysische und experimentelle Natur des epistemischen Unternehmens, siehe dazu beispielsweise Ernst Mach (1838-1916).

14 Der Paläontologe Nicholson merkte in einem Aufsatz über den Fortschritt in der paläontologischen Forschung an, dass der ,first point [...] which demands our attention is the immense benefit which has resulted to palaeontology from the introduction of the microscope as an absolutely indispensable instrument of palaeontological research. The old ,macroscopic' method of investigation, as the Germans have happily termed it, has been definitely abandoned; and no palaeontologist would now, except under special circumstances, describe any doubtful or problematical Invertebrate fossil without previously having subjected it to a rigid examination by means of the microscope, at any rate in all cases in which his specimens would allow of this mode of examination. It is impossible, indeed, to exaggerate the immensely increased powers of observation and description which paleontologists have acquired by the practice of making transparent sections of fossils, suitable for microscopic examination. Already, the results of this method of inquiry have proved extremely important, and when its adoption has become more universal, we may expect an even more remarkable increase of our knowledge as to the intimate structure of many extinct forms of life, which are at present but very imperfectly understood." (Nicholson 1878: 5)

15 Diese Hauptaufgabe wurde von der Deszendenztheorie eingeführt: „Der Forschung zeigt die Descendenztheorie schon im Voraus die Richtung an, in welcher die erfolgreichsten Entdeckungen zu machen sind; sie zwingt zu einer festen Methode der Untersuchung" (Zittel 1876a: 42).

16 Unter natürlicher Systematik verstand Zittel eine reine biologische und deshalb nicht geologische Systematik.

17 Wie Lynn Nyhart feststellte, nutzten Ende des 19. Jahrhunderts die meisten deutschen Zoologen genau diese Methode: „Research in comparative anatomy in turn became almost exclusively the concern of zoologists, who continued to apply it to problems of phylogeny, but usually without the primary interest in form that had characterized earlier [...] most German zoologists were occupied with elaborating taxonomies, on the one hand, and, on the other, focusing much more directly on problems with the Darwinian mechanisms of evolution" (Nyhart 1987: 389). Siehe auch Nyhart 1995.

18 „Darwin hat es zuerst betont und nach ihm haben Haeckel und Andere oft hervorgehoben, dass die Systematik durch die neuen Gesichtspunkte, welche die Descendenztheorie eröffnet, eine ganz durchgreifende Veränderung erfahren muss und dass dieses an sich trockene Feld ungeahntes Interesse gewinnt, wenn die Systematik sich bestrebt, auf genetischer Basis sich umzugestalten, die Descendenzbeziehungen zur Darstellung und zum Ausdruck zu bringen; in ganz hervorragendem Maasse gilt dies von der Paläontologie." (Neumayr 1874: 144). Der Paläontologe Othenio Abel (1875-1946) nutzte die Phylogenese ebenso im trockenen Feld der Systematik (Abel 1914).

19 Wilhelm Heinrich Waagen (1841-1900) führte den modernen Begriff der Formenreihe in die paläontologische Praxis ein. Er beschrieb die Formenreihen als eine Praxis, womit man die „Bildungstypen oft durch eine grosse Zahl von Schichten hindurch verfolgen [kann], aber in jeder Schicht zeigen die Individuen eine von den vorhergehenden und nachfolgenden etwas abweichende Gestalt; das Ganze bildet eine zusammenhängende Reihe“ (Waagen 1869: 185). Zittel schrieb deshalb: „Je mehr sich das palaeontologische Material vergrössert, desto zahlreicher und vollständiger werden die Formenreihen“ (Zittel 1895b). Nach Neumayr weisen die Formenreihen die allmähliche und schrittweise Veränderung der Organismen, d.h. den darwinistischen Mechanismus der Evolution (Neumayr 1886; 1889), nach.

20 So schrieb beispielsweise Henrik Steffens (1773-1845): „Hier ist das Buch, sagte er dann, und gab uns den Linné; die Pflanze ist hier beschrieben, hier muß sie aufgesucht werden, Kinder amüsieren sich mit Bildern“ (Steffens 1840: 312). 
21 Wie ich bereits in Anmerkung 10 erwähnte, ermöglicht Zittels Fossilbegriff wichtige Fragestellungen zum Begriff der paläontologischen Repräsentation und Sichtbarkeit, die in diesem Aufsatz nur am Rande erwähnt werden können.

22 Es sei angemerkt, dass die Zeitschrift Palaeontolographica unter Zittels Führung detaillierte Abbildungen der Fossilien einführte.

23 Wie Nicholson schrieb, ,the few thousand years of which we have historical evidence sink into absolute insignificance beside the unnumbered aeons which unroll themselves one by one as we penetrate the dim recesses of the past. [...] Even speculation droops her wings in the attenuated atmosphere of a past so remote, and the light of imagination is quenched in the darkness of a history so ancient" (Nicholson 1877: 9).

\section{Literatur}

Abel, Othenio, 1914. Die Stämme der Wirbeltiere. Berlin, Leipzig: De Gruyter \& Co.

Ager, Derek, 1983. Palaeontology by the Telephone Book. Nature, 304 (4), 470.

Beringer, Carl Christoph, 1954. Geschichte der Geologie und des Geologischen Weltbildes. Stuttgart: Ferdinand Enke.

Bischoff, Gottlieb Wilhelm/Blum, Johann Reinhard/Bronn, Heinrich Georg/von Leonhard, Cäsar C./Leuckart, Friedrich Sigismund/Voigt, Friedrich Sigmund, 1841. Naturgeschichte der drei Reiche. Stuttgart: Schweizerbart'sche Verlagsbuchhandlung.

Blum, Ann Shelby, 1993. Picturing Nature. American Nineteenth-Century Zoological Illustration. Princeton: Princeton University Press.

Breidenmoser, Tobias/Engler, Fynn Ole/Jirikowski, Günther/Pohl, Michael/Weiss, Dieter G., 2010. Transformation of Scientific Knowledge in Biology: Changes in our Understanding of the Living Cell Through Microscopic Imaging. Berlin: MPIWG Preprint 408.

Bronn, Heinrich Georg, 1841-1849. Handbuch einer Geschichte der Natur. Stuttgart: Schweizerbart'sche Verlagsbuchhandlung.

Bronn, Heinrich Georg, 1849a. Einige Betrachtungen über paläontologische Statik bearbeitet nach der "Geschichte der Natur". Neues Jahrbuch für Mineralogie, Geognosie, Geologie, und Petrefakten-Kunde

Bronn, Heinrich Georg, 1849b. Index palaeontologicus oder Übersicht der bis jetzt bekannten fossilen Organismen. Stuttgart: Schweizerbart'sche Verlagsbuchhandlung.

Bronn, Heinrich Georg, 1858. Untersuchungen über die Entwickelungs-Gesetze der organischen Welt während der Bildungs-Zeit unserer Erd-Oberfläche. Stuttgart: Schweizerbart'sche Verlagsbuchhandlung.

Crary, Jonathan, 1988. Techniques of Observer. On Vision and Modernity the Nineteenth Century. Cambridge: The MIT Press.

Daston, Lorraine, 1998. Fear and Loathing of the Imagination in Science. Daedalus, 127 (1), 73-95.

Daston, Lorraine, 2014. Beyond Representation. In: Wiebe E. Bijker, W. Bernard Carlson und Trevor Pinch, Hg., Representation in Scientific Practice Revisited. Cambridge: The MIT Press.

Daston, Lorraine/Galison, Peter, 1992. The Image of Objectivity. Representations, 40 (1), 81-128.

Daston, Lorraine/Galison, Peter, 2007. Objectivity. Brooklyn, NY: Zone Books.

Dupré, Sven, 2010. Art History, History of Science, and Visual Experience. Isis, 101 (3), 618-622.

Eldredge, Niles/Gould, Stephen Jay, 1972. Punctuated Equilibria: An Alternative to Phyletic Gradualism. In: Thomas J. M. Schopf, Hg., Models in Paleobiology. San Francisco: Freeman, Cooper and Co.

Farber, Paul Lawrence 2000. Finding Order in Nature: The Naturalist Tradition From Linnaeus To E. O. Wilson. Baltimore: Johns Hopkins University Press.

Golinski, Jan, 1998. Making Natural Knowledge. Constructivism and History of Science. Cambridge: Cambridge University Press.

Gould, Stephen Jay, 1980. The Promise of Paleontology as a Nomothetic, Evolutionary Discipline. Paleobiology, 6 (1), 96-118.

Gould, Stephen Jay, 1989. Wonderful Life: The Burgess Shale and the Nature of History. New York: Norton \& Co. 
Gould, Stephen Jay/Eldredge, Niles, 1977. Punctuated Equilibria: The Tempo and Mode of Evolution Reconsidered. Paleobiology, 3 (2), 115-151.

Greene, Mott T., 1982. Geology in the Nineteenth Century: Changing Views of a Changing World. Ithaca: Cornell University Press.

Haeckel, Ernst, 1866. Generelle Morphologie der Organismen: allgemeine Grundzüge der organischen Formen-Wissenschaft, mechanisch begründet durch die von Charles Darwin reformirte Descendenz-Theorie. Berlin: Reimer.

Heigel, K. Th. von 1904. Zum Andenken an Karl von Zittel. München: Verlag der K. B. Akademie.

Hentschel, Klaus, 2014. Visual Cultures in Science and Technology. A Comparative History. Oxford: Oxford University Press.

Heumann, Ina/Hüntelmann, Axel C., 2013. Einleitung: Bildtatsachen. Visuelle Praktiken der Wissenschaften. Berichte zur Wissenschaftsgeschichte, 36 (4), 283-293.

Hölder, Helmut, 1989. Kurze Geschichte der Geologie und Paläontologie. Berlin; Heidelberg: Springer.

Jaekel, Otto, 1891. Ueber mikroskopische Untersuchungen im Gebiet der Palaeontologie. Neues Jahrbuch für Mineralogie, Geognosie, Geologie und Petrefaktenkunde

Kulvicki, John, 2010. Knowing with Images: Medium and Messages. Philosophy of Science, 77 (2), 295-313.

Larson, James, 1994. Interpreting Nature: The Science of Living Form from Linnaeus to Kant. Baltimore: Johns Hopkins University Press.

Lehmann, Ulrich, 1983. Fossil invertebrates. Cambridge: Cambridge University Press.

Lepenies, Wolf, 1976. Das Ende der Naturgeschichte: Wandel kultureller Selbstverständlichkeiten in den Wissenschaften des 18. und 19. Jahrhunderts. München: Hanser.

Maynard Smith, John, 1984. Palaeontology at the High Table. Nature, 309 (5967), 401-402.

Neumayr, Melchior, 1874. Die Fauna der Schichten mit Aspidoceras acanthicum. Abhandlungen der Geologischen Bundesanstalt in Wien, 141-257.

Neumayr, Melchior, 1895. Erdgeschichte. Leipzig; Wien: Verlag des Bibliographischen Instituts.

Nicholson, Henry Alleyne, 1877. The Ancient Life-History of the Earth. Edinburgh London: William Blackwood and Sons

Nicholson, Henry Alleyne, 1878. Recent Progress in Palaeontology. The Geological Magazine, V (1), 1-13.

Nyhart, Lynn K., 1987. The Disciplinary Breakdown of German Morphology, 1870-1900. Isis, 78 (3), 365-389.

Nyhart, Lynn K., 1995. Biology Takes Form. Animal Morphology and the German Universities 1800-1900. Chicago: The University of Chicago Press.

Oppel, Albert, 1856-1858. Die Juraformation Englands, Frankreichs und des südwestlichen Deutschlands, nach ihren einzelnen Gliedern eingetheilt und verglichen. Jahreshefte des Vereins für vaterländische Naturkunde in Württemberg, 12-14 (121-556; 141-396; 129291).

Osborn, Henry Fairfield, 1904. Karl Alfred von Zittel. Science, 19 (474), 186-188.

Porter, Theodore M., 1995. Trust in Numbers: The Pursuit of Objectivity in Science and Public Life. Princeton: Princeton University Press.

Quenstedt, Friedrich August, 1852. Handbuch der Petrefaktenkunde Tübingen: Laupp.

Quenstedt, Friedrich August 1858. Der Jura. Tübingen: Laupp.

Rainger, Ronald, 1981. The Continuation of the Morphological Tradition: American Paleontology, 1880-1910. Journal of the History of Biology, 14 (1), 129-158.

Rainger, Ronald, 1991. An Agenda for Antiquity: Henry Fairfield Osborn \& Vertebrate Paleontology at the American Museum of Natural History, 1890-1935. Tuscaloosa: University of Alabama Press.

Raup, David M./Sepkoski, J. John., Jr., 1984. Periodicity of Extinctions in the Geologic Past. Proceedings of the National Academy of Sciences USA, 81 (3),

Raup, David M./Stanley, Steven M., 1971. Principles of Paleontology. San Francisco: Freeman.

Reif, Wolf-Ernst, 1986. The Search for a Macroevolutionary Theory in German Paleontology. Journal of the History of Biology, 19 (1), 79-103.

Rheinberger, Hans-Jörg/Hagner, Michael/Schmidt-Wahrig, Bettina, 1997. Räume des Wissens. Repräsentation, Codierung, Spur. In: Hans-Jörg Rheinberger, Michael Hagner und Bettina Schmidt-Wahrig, Hg., Räume des Wissens. Repräsentation, Codierung, Spur. Berlin: Akademie-Verlag. 
Romer, Alfred Sherwood, 1933. Vertebrate Paleontology. Chicago: The University of Chicago Press.

Rudwick, Martin J. S, 1985. Scenes From Deep Time: Early Pictorial Representations of the Prehistoric World. Chicago: The University of Chicago Press.

Rudwick, Martin J. S, 2005. Bursting the Limits of Time: The Reconstruction of Geohistory in the Age of Revolution. Chicago: The University of Chicago Press.

Rudwick, Martin J. S, 2008. Worlds Before Adam: The Reconstruction of Geohistory in the Age of Reform. Chicago: The University of Chicago Press.

Schlich, Thomas, 1995. „Wichtiger als der Gegenstand selbst“: Die Bedeutung des fotografischen Bildes in der Begründung der bakteriologischen Krankheitsauffassung durch Robert In: Thomas Schlich und Martin Dinges, Hg., Neue Wege in der Seuchengeschichte. Stuttgart: Steiner.

Schrock, Robert R./Twenhofel, William H., 1953. Principles of Invertebrate Paleontology. New York; Toronto; London: McGraw-Hill Book Company, Inc.

Schumacher, Ingrid 1975. Die Entwicklungstheorie des Heidelberger Paläontologen und Zoologen Heinrich Georg Bronn (1800-1862). Heidelberg: Ruprecht-Karls-Universität.

Sepkoski, David, 2012. Rereading the Fossil Record: The Growth of Paleobiology as an Evolutionary Discipline. Chicago: The University of Chicago Press.

Sepkoski, David, 2013. Towards „A Natural History of Data“: Evolving Practices and Epistemologies of Data in Paleontology, 1800-2000. Journal of the History of Biology, 46 (3), 401-444.

Sepkoski, David/Ruse, Michael, 2009. The Paleobiological Revolution: Essays on the Growth of Modern Paleontology. Chicago: The University of Chicago Press.

Sepkoski, J. John Jr., 1982. A Compendium of Fossil Marine Families. Milwaukee: Milwaukee Public Museum.

Sepkoski, J. John., Jr., 1978. A Kinetic Model of Phanerozoic Taxonomic Diversity. I. Analysis of Marine Orders. Paleobiology, 1 (4), 223-251.

Sepkoski, J. John., Jr., 1994. What I Did with My Research Career. In: William Glen, Hg., MassExtinction Debates: How Science works in a Crisis. Stanford: Stanford University Press.

Sepkoski, J. John., Jr./Bambach, Richard/Raup, David M./Valentine, James W., 1981. Phanerozoic Marine Diversity and The Fossil Record. Nature, 293 (5832), 435-437.

Steffens, Henrik, 1840. Was ich erlebte. Aus der Erinnerung niedergeschrieben. Breslau: Josef Max und Komp.

Tamborini, Marco, 2015. Paleontology and Darwin's Theory of Evolution: The Subversive Role of Statistics at the End of the 19th Century. Journal of the History of Biology, Online First (http:// link.springer.com/article/10.1007\%2Fs10739-015-94.02-y.)

Tamborini, Marco, Under Review. What Did it Mean to be a Darwinian Palaeontologist? The Importance of Darwin in German Palaeontology at the End of the 19th Century As a Case of Pyrrhic Victory.

Turner, Derek, 2011. Paleontology a Philosophical Introduction. Cambridge; New York: Cambridge University Press.

Valentine, James W., 1969. Patterns of Taxonomic and Ecological Structure of the Shelf Benthos During Phanerozoic Time. Paleontology, 12 (4), 684-709.

Waagen, Wilhelm Heinrich, 1869. Die Formenreihe des Ammonites subradiatus: Versuch einer paläontologischen Monographie. Geogn.-paläontol. Beiträge

Wagenbreth, Otfried, 1999. Geschichte der Geologie in Deutschland. Stuttgart: Georg Thieme Verlag.

Wilson, Catherine, 1995. The Invisible World: Early Modern Philosophy and the Invention of the Microscope. Princeton: Princeton University Press.

Ziegler, Bernhard, 1984. Kleine Geschichte der Paläontologie. Stuttgart: Staatl. Museum für Naturkunde.

Zittel, Karl Alfred von, 1871. Aus der Urzeit. München: Rudolph Oldenbourg.

Zittel, Karl Alfred von, 1876a. Handbuch der Palaeontologie (Palaeozoologie). München; Leipzig: Rudolph Oldenbourg.

Zittel, Karl Alfred von, 1876b. Ueber Coeloptychium. Ein Beitrag zur Kenntniss der Organisation fossiler Spongien. Abhandlungen der Mathemat.-physikal. Classe der Königlich Bayerischen Akademie der Wissenschaften, 12-13 (1-80).

Zittel, Karl Alfred von, 1895. Grundzüge der Paläontologie (Paläozoologie). München: Rudolph Oldenbourg. 
Zittel, Karl Alfred von, 1897. Ontogenie, Phyllogenie und Systematik. In: Anonymous, Congrès Géologique International. Compte-Rendu de la Sixième Session, en Suisse. Lausanne: Imprimerie Georges Bridel.

Zittel, Karl Alfred von, 1899. Geschichte der Geologie und Paläontologie bis Ende des 19. Jahrhunderts. München: Rudolph Oldenbourg.

Zittel, Karl Alfred von, 1902. Ueber wissenschaftliche Wahrheit. Rede in der oeffentlichen Festsitzung der Akademie am 15. November 1902. München: Verlag der k. b. Akademie.

Marco Tamborini

PAN, Perspektiven auf Natur

Leibniz-Institut für Evolutions- und

Biodiversitätsforschung

Museum für Naturkunde

Invalidenstraße 43

10115 Berlin

Germany

E-Mail: marco.tamborini@mfn-berlin.de 